\title{
Nasal Oxytocin Administration Reduces Food Intake without Affecting Locomotor Activity and Glycemia with c-Fos Induction in Limited Brain Areas
}

\author{
Yuko Maejima a, b Rauza Sukma Rita ${ }^{a}$ Putra Santoso ${ }^{a}$ Masato Aoyamac \\ Yuichi Hiraoka $^{d}$ Katsuhiko Nishimori ${ }^{d}$ Darambazar Gantulga ${ }^{a}$ \\ Kenju Shimomura ${ }^{a}$ b Toshihiko Yada a, e \\ a Division of Integrative Physiology, Department of Physiology, Jichi Medical University School of Medicine, \\ Shimotsuke, ${ }^{\mathrm{b}}$ Department of Electrophysiology and Oncology, Fukushima Medical University School of Medicine, \\ Fukushima, 'Department of Animal Science, Faculty of Agriculture, Utsunomiya University, Utsunomiya, \\ ${ }^{d}$ Department of Molecular and Cell Biology, Graduate School of Agricultural Science, Tohoku University, Miyagi, \\ and ${ }^{e}$ Division of Adaptation Development, Department of Developmental Physiology, National Institute for \\ Physiological Sciences, Okazaki, Japan
}

\section{Key Words}

Oxytocin · Nasal treatment · Paraventricular nucleus · Dorsal motor nucleus of vagus · Glucose tolerance - Obesity ·

Insulin release

\begin{abstract}
Recent studies have considered oxytocin (Oxt) as a possible medicine to treat obesity and hyperphagia. To find the effective and safe route for Oxt treatment, we compared the effects of its nasal and intraperitoneal (IP) administration on food intake, locomotor activity, and glucose tolerance in mice. Nasal Oxt administration decreased food intake without altering locomotor activity and increased the number of c-Fos-immunoreactive (ir) neurons in the paraventricular nucleus (PVN) of the hypothalamus, the area postrema (AP), and the dorsal motor nucleus of vagus (DMNV) of the medulla. IP Oxt administration decreased food intake and locomotor activity and increased the number of c-Fos-ir neurons not only in the PVN, AP, and DMNV but also in the nucleus of
\end{abstract}

solitary tract of the medulla and in the arcuate nucleus of the hypothalamus. In IP glucose tolerance tests, IP Oxt injection attenuated the rise of blood glucose, whereas neither nasal nor intracerebroventricular Oxt affected blood glucose. In isolated islets, Oxt administration potentiated glucose-induced insulin secretion. These results indicate that both nasal and IP Oxt injections reduce food intake to a similar extent and increase the number of c-Fos-ir neurons in common brain regions. IP Oxt administration, in addition, activates broader brain regions, reduces locomotor activity, and affects glucose tolerance possibly by promoting insulin secretion from pancreatic islets. In comparison with IP administration, the nasal route of Oxt administration could exert a similar anorexigenic effect with a lesser effect on peripheral organs. 


\section{Introduction}

Oxytocin (Oxt) is a neurohypophysial hormone that regulates uterine contraction during labor and milk ejection [1]. Oxt is synthesized in the paraventricular (PVN) and supraoptic nucleus of the hypothalamus. It is released peripherally after being shuttled to the pituitary [2], or centrally in the brain to regulate neuronal process [3]. Recent studies have clarified new functions of Oxt in the central nervous system, including increased trust [4], regulation of social recognition [5] and development of mother-infant bonding [6]. In addition, our previous study employed Oxt in the PVN-driven anorexigenic circuit in rats [7].

The anorexigenic effect of Oxt has been reported since the early 1990s. Olson et al. [8] showed that Oxt and its agonist decrease food intake when administered centrally in rats, and Arletti et al. [9] showed that intracerebroventricular (icv) injection of Oxt inhibits food intake together with water intake. More recent studies have shown that mice with deficient Oxt or Oxt receptor (Oxt-R) develop late-onset obesity $[10,11]$.

Obesity is linked to numerous diseases including type 2 diabetes, cardiovascular events, and certain forms of cancer $[12,13]$. Several antiobesity drugs, including diethylpropion, fenproporex, mazindol, fluoxetine, and sibutramine, have been developed [14], but there is little evidence for effective and safe treatments.

We have previously shown that subchronic peripheral Oxt treatment through a subcutaneously implanted osmotic minipump reduces hyperphagia and obesity in high-fat diet-fed obese mice, and that intraperitoneal (IP) Oxt treatment similarly reduces hyperphagia [15]. Zhang et al. [16] have recently reported that the nasal administration of Oxt decreases body weight (BW) in obese subjects. Ott et al. [17] showed that nasal Oxt treatment reduces reward-driven food intake. These reports indicate that subcutaneous, IP, and nasal routes of Oxt treatment have common actions of reducing obesity and hyperphagia. However, a comparison of these actions and underlying mechanisms following different administration routes of Oxt has not been previously undertaken.

We performed a comparative study on the effects of nasal and IP administration of Oxt on feeding, locomotor activity, and glucose tolerance in mice, and found that both nasal and IP Oxt injections reduce food intake to a similar extent with increased c-Fos-immunoreactive (ir) neurons in common brain regions. IP Oxt administration also increased c-Fos-ir neurons in additional brain re- gions, reduced locomotor activity, and affected glucose tolerance possibly by promoting insulin secretion from pancreatic islets.

\section{Materials and Methods}

\section{Animals}

Male C57BL/6J mice (aged 6 weeks) were obtained from Japan SLC (Hamamatsu, Japan). Animals were maintained on a 12-hour light/dark cycle. The dark and light phases started at 19:30 h and 7:30 h, respectively. Mice were allowed ad libitum access to water and a standard diet (CE-2; Clea, Osaka, Japan). Experimental procedures and care of animals were carried out according to the Jichi Medical University Institute of Animal Care and Use Committee. The mice used in the experiments were well habituated to minimize stress.

\section{Measurements of Food Intake and Locomotor Activity after} Oxt Administration

Following deprivation of food for $2 \mathrm{~h}$ before the dark phase, the animals received $0.1-10 \mu \mathrm{g} / 10 \mu \mathrm{l}$ nasal Oxt or 40 and $400 \mu \mathrm{g} /$ kg IP Oxt (Peptide Institute, Osaka, Japan) 30 min before the dark phase. For the nasal administration, $10 \mu \mathrm{l}$ of Oxt or vehicle was dropped into the nasal cavity. Cumulative food intake was measured for the following $0.5,1,2,6$, and $24 \mathrm{~h}$. For the measurements during the dark phase, red light with minimum intensity was used to prevent mice from being stimulated by bright light. Locomotor activity was measured by an activity monitoring system (ACTIMO-100; Shinfactory, Fukuoka, Japan). The doses of nasal Oxt were $0.1,1$ and $10 \mu \mathrm{g}$, based on the minimum effective dose of Oxt $(1 \mu \mathrm{g})$ on food intake determined by pilot experiments. The dose of IP Oxt was based on a previous report [15] showing that $400 \mu \mathrm{g} / \mathrm{kg}$ Oxt markedly and long-lastingly suppressed food intake.

\section{Immunostaining of c-Fos after Oxt Administration}

Food was deprived for $2 \mathrm{~h}$ before the dark phase until perfusion. The animals received nasal $(1 \mu \mathrm{g} / 10 \mu \mathrm{l})$ or IP Oxt $(400 \mu \mathrm{g} / \mathrm{kg}) \mathrm{ad}-$ ministration $30 \mathrm{~min}$ before the dark phase. After $90 \mathrm{~min}$, the mice were transcardially perfused as described previously [7]. Coronal sections of $40-\mu \mathrm{m}$ thickness were cut with a freezing microtome. Sections at $120-\mu \mathrm{m}$ intervals between -0.58 and $-8.0 \mathrm{~mm}$ from the bregma were used for c-Fos immunohistochemistry. Sections were rinsed in phosphate-buffered saline (PBS; $0.01 \mathrm{M}, \mathrm{pH} 7.4$ ), incubated in PBS containing $2 \%$ normal goat serum and $2 \%$ bovine serum albumin (BSA), then incubated with rabbit anti-c-Fos antiserum (sc-52, 1:5,000; Santa Cruz, Calif., USA). Subsequently, the sections were incubated with biotinylated goat anti-rabbit IgG (1:500; Vector Laboratories Inc., Calif., USA), and an avidin-biotin complex (ABC kit; Vector Laboratories Inc.). Immunoreactions were visualized by incubating in diaminobenzidine (DAB) solution containing nickel ammonium.

Bilateral sections of the hypothalamus and brainstem between -0.58 and $-8.0 \mathrm{~mm}$ from the bregma were prepared, with c-Fos-ir cells per section counted manually under the microscope. The cell count per section was averaged for all sections in each investigated nucleus of each animal.
Maejima/Rita/Santoso/Aoyama/Hiraoka/ Nishimori/Gantulga/Shimomura/Yada 
Glucose Tolerance Test and Measurement of Plasma Insulin

Mice aged 10 weeks (or with a BW of 22-25 g) were cannulated in the lateral ventricle $(0.5 \mathrm{~mm}$ caudal to bregma, $1.0 \mathrm{~mm}$ lateral from the midline, and $2.2 \mathrm{~mm}$ below the skull surface; ICM-23G09 Inter Medical, Osaka, Japan) and housed individually. BW and food intake were not significantly different before the operation and for 10 days thereafter. On the day of the experiment, food was deprived at 09:00 $\mathrm{h}$ and an IP glucose tolerance test (IPGTT; $2 \mathrm{~g} / \mathrm{kg}$ ) was started at 13:00 h. IPGTT was performed 20 min after nasal $(0.1,1,10 \mu \mathrm{g})$, icv $(0.4,4 \mu \mathrm{g})$ or IP $(40,400 \mu \mathrm{g} / \mathrm{kg})$ Oxt injection. Oxt doses for icv injection were determined based on our previous report [7]. Blood was sampled by cutting the surface of the tail skin under local anesthesia with EMLA cream (AstraZeneca K.K., London, UK) without restraint. Blood glucose levels were measured using Glucocard (Arkray, Kyoto, Japan).

For the measurement of plasma insulin, mice aged 10 weeks (22-26 g BW) were used. IP Oxt injection $(400 \mu \mathrm{g} / \mathrm{kg})$ and IPGTT procedures followed the same protocol and time course. The mice were decapitated, and their blood was collected $10 \mathrm{~min}$ after IPGTT. After centrifugation, plasma insulin concentration was measured by ultrasensitive mouse insulin ELISA kit (Morinaga Institute of Biological Science, Inc., Yokohama, Japan).

\section{Isolation of Islets and Measurement of Insulin Secretion}

Mice with similar age and BW to those in the IPGTT were used. Islets were isolated as previously described [18] and cultured overnight in DMEM solution (Sigma, St. Louis, Mo., USA). After 30 min of preincubation in $2 \mathrm{mM}$ glucose solution and $30 \mathrm{~min}$ under static incubation conditions in Krebs-Ringer Buffer (mM): 120 $\mathrm{NaCl}, 4.7 \mathrm{KCl}, 2.5 \mathrm{CaCl}_{2}, 1 \mathrm{KH}_{2} \mathrm{PO}_{4}, 1.2 \mathrm{MgSO}_{4}, 10 \mathrm{HEPES}, 25$ $\mathrm{NaHCO}_{3}$, pH 7.4 (with $\mathrm{NaOH}$ ), plus $0.1 \%$ BSA, experimental glucose $(2,5,20 \mathrm{mM})$ with or without Oxt $(10 \mathrm{nM})$, insulin secretion was measured. Islets were then incubated overnight at $-20^{\circ} \mathrm{C}$ with acidified ethanol solution (95\% ethanol, 5\% acetic acid) to extract all insulin. Insulin was measured using a Morinaga mouse ELISA kit (Morinaga Institute of Biological Science, Inc.).

\section{Statistical Analysis}

All data are presented as means \pm SEM. The statistical analysis of experimental data of c-Fos-ir neurons, insulin secretion, and plasma insulin were carried out using Student's t test. Data for food intake, locomotor activity, and glucose tolerance test were analyzed by repeated measures of two-way ANOVA with treatment (saline vs. Oxt including dose responses) and time as factors. Post hoc multiple comparisons were made using Tukey's test. Significance was set at $\mathrm{p}<0.05$ for all analyses.

\section{Results}

\section{Effect of Oxt Administration on Food Intake and \\ Locomotor Activity}

In order to compare the effects of nasal and IP Oxt administration, we administered an approximately equally effective amount of Oxt for nasal and IP administration (nasal: $0.1-10 \mu \mathrm{g} / 25 \mathrm{~g} \mathrm{BW}$, i.e. approx. $4-400$ $\mu \mathrm{g} / \mathrm{kg}$; IP: $40-400 \mu \mathrm{g} / \mathrm{kg}$ ). Nasal administration of Oxt at doses of $0.1-10 \mu \mathrm{g} / 10 \mu \mathrm{l}$ was performed in mice weighing 20-25 g. These doses correspond to approximately 4$400 \mu \mathrm{g} / \mathrm{kg}$.

Nasal administration of $0.1 \mu \mathrm{g}$ Oxt did not affect the food intake significantly $\left(\mathrm{F}_{1,60}=2.53, \mathrm{p}>0.05\right.$; fig. 1a). Administration of $1 \mu \mathrm{g}$ Oxt significantly decreased the food intake $\left(\mathrm{F}_{1,56}=27.4, \mathrm{p}<0.01\right.$; fig. $\left.1 \mathrm{~b}\right)$, and $10 \mu \mathrm{g}$ Oxt $\left(\mathrm{F}_{1,92}=27.16, \mathrm{p}<0.01\right.$; fig. $\left.1 \mathrm{c}\right)$. Tukey's test indicated that nasal administration of $1 \mu \mathrm{g}$ Oxt reduced the cumulative food intake 6 and $24 \mathrm{~h}$ after administration (fig. 1b), and $10 \mu \mathrm{g}$ Oxt reduced the food intake 0.5, 1, 2, 6, and $24 \mathrm{~h}$ after administration (fig. 1c) without changing locomotor activity $\left(\mathrm{F}_{1,299}=0.10, \mathrm{p}>0.05\right.$; fig. $\left.2 \mathrm{a}, \mathrm{c}\right)$.

IP administration of $40 \mu \mathrm{g} / \mathrm{kg}$ Oxt failed to significantly affect the food intake $\left(\mathrm{F}_{1,24}=2.27, \mathrm{p}>0.05\right.$; fig. $\left.1 \mathrm{~d}\right)$ and $400 \mu \mathrm{g} / \mathrm{kg}$ Oxt markedly decreased the food intake at 0.5 , 1,2 , and $6 \mathrm{~h}\left(\mathrm{~F}_{1,24}=38.9, \mathrm{p}<0.01\right.$; fig. $\left.1 \mathrm{e}\right)$ but not at $24 \mathrm{~h}$. IP injection of $400 \mu \mathrm{g} / \mathrm{kg}$ Oxt showed a tendency to decrease locomotor activity $\left(\mathrm{F}_{1,138}=2.6, \mathrm{p}>0.05\right)$ for the first $7 \mathrm{~h}$ and significantly decreased cumulative locomotor activity during the dark phase (fig. $2 b, d$ ).

The tendency and time course of reduction in the food intake were similar between nasal and IP administration (fig. 1b, d). However, at a higher dose (approx. $400 \mu \mathrm{g} /$ $\mathrm{kg})$, nasal administration of Oxt $(10 \mu \mathrm{g} / \mu \mathrm{l}$; fig. $1 \mathrm{c})$ reduced the cumulative food intake for up to $24 \mathrm{~h}$, but the extent of food intake reduction during $0.5-6 \mathrm{~h}$ was smaller compared to IP administration (fig. 1e).

\section{Effect of Oxt Administration on the Number of $c$-Fos-ir Neurons}

After nasal administration of $1 \mu \mathrm{g}$ Oxt, the number of c-Fos-ir neurons was significantly increased in the PVN of the hypothalamus. The number was $137.1 \pm 25.6 / \mathrm{sec}-$ tion in controls versus $284.6 \pm 37.4 /$ section in Oxt-injected groups ( $\mathrm{p}<0.05$; fig. $3 \mathrm{a}, \mathrm{b}, \mathrm{e})$. Nasal Oxt administration also increased the number of c-Fos-ir neurons in the medulla, the area postrema (AP; $10.6 \pm 3.5 /$ section in controls vs. $27.3 \pm 4.7 /$ section in Oxt-injected groups), and the dorsal motor nucleus of vagus (DMNV; $32.3 \pm 6.9 /$ section in controls vs. $69.2 \pm 4.7 /$ section in Oxt-injected groups; fig. 3c-e). IP administration of Oxt also increased the number of c-Fos-ir neurons in the PVN $(71.7 \pm 18.1$ / section in controls vs. $188.0 \pm 35.2 /$ section in Oxt-injected groups), DMNV (14.7 $\pm 3.7 /$ section in controls vs. 43.4 \pm 4.8 /section in Oxt-injected groups; fig. $3 \mathrm{f}, \mathrm{g}, \mathrm{j}$ ), nucleus of the solitary tract (NTS; $39.5 \pm 9.8 /$ section in controls vs. $153.3 \pm 6.0$ /section in Oxt-injected groups), and the arcuate nucleus (ARC; $68.9 \pm 12.2$ /section in control vs. $128.6 \pm 7.0$ in Oxt groups), consistent with our previous 

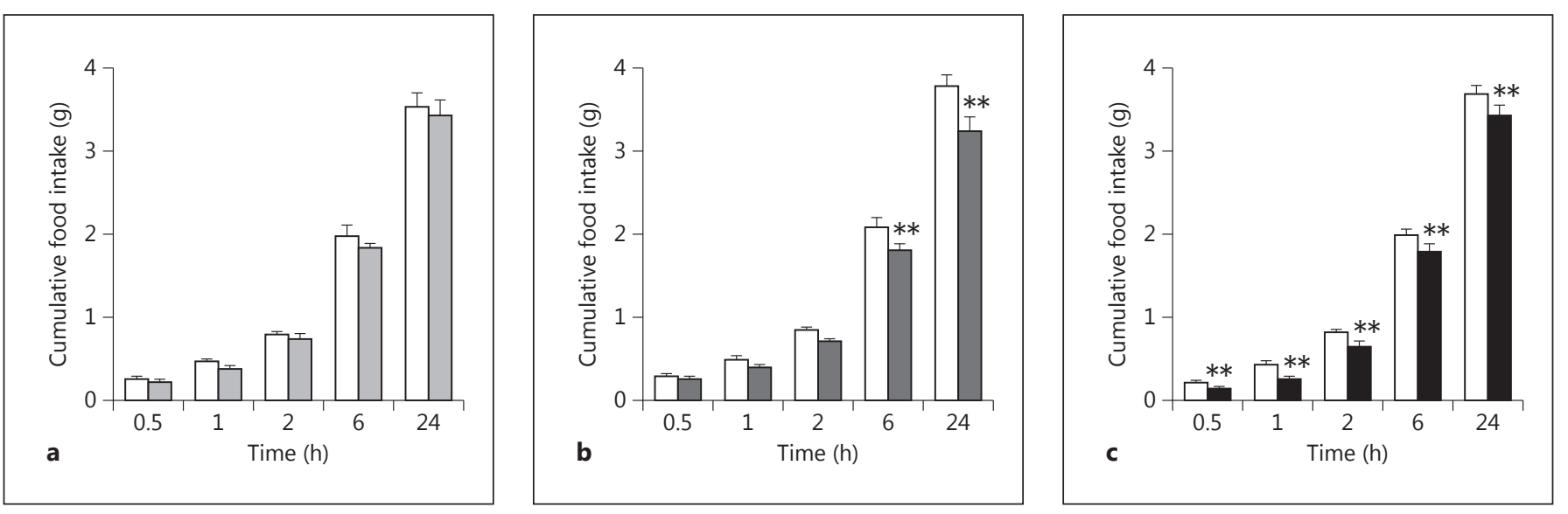

Fig. 1. Effects of nasal and IP injections of Oxt on food intake in mice. a-c Food intake after nasal administration of $0.1 \mu \mathrm{g}$ Oxt (a; n = 8-9), $1 \mu \mathrm{g}$ Oxt (b; n = 7-9) and $10 \mu \mathrm{g}$ Oxt (c; $\mathrm{n}=12-13)$. The white and filled bars indicate the saline- and Oxt-administered groups, respectively. d, e Food intake after IP injection of $40 \mu \mathrm{g} / \mathrm{kg}$ Oxt (d; $\mathrm{n}=4)$ and $400 \mu \mathrm{g} / \mathrm{kg}$ Oxt (e; $\mathrm{n}=4)$. Differences were assessed by repeated measures two-way ANOVA with Oxt treatment and time as factors. Post hoc multiple comparisons were made using Tukey's test. ${ }^{*} \mathrm{p}<$ $0.05,{ }^{* *} \mathrm{p}<0.01$.

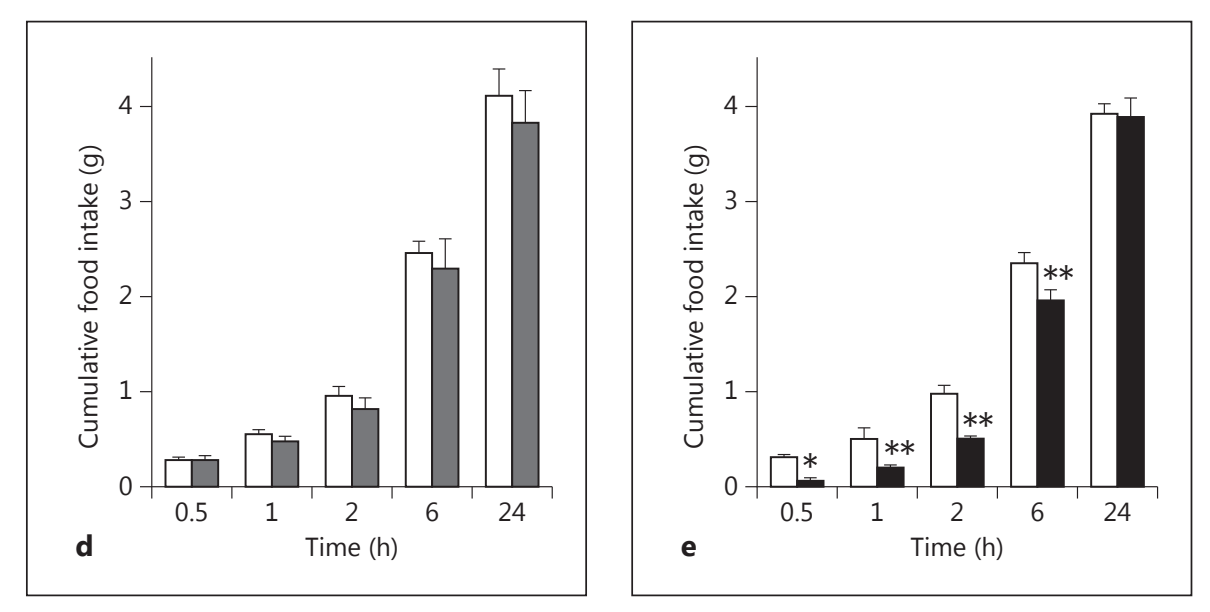

report [15]. Thus, IP but not nasal administration of Oxt increased c-Fos-ir neurons in the NTS and ARC.

\section{Effect of Oxt Administration on Glucose Metabolism}

The effect of Oxt on the elevated levels of blood glucose during IPGTT was examined. Nasal and icv Oxt administration had no effect on blood glucose levels in IPGTT $\left(\mathrm{F}_{3,84}=0.28, \mathrm{p}>0.05\right.$, and $\mathrm{F}_{2,54}=1.02, \mathrm{p}>0.05$, respectively; fig. $4 \mathrm{a}, \mathrm{c})$. In contrast, IP administration of 40 and $400 / \mu \mathrm{g} / \mathrm{kg}$ Oxt $\left(\mathrm{F}_{2,36}=13.10, \mathrm{p}<0.01\right)$ dose-dependently attenuated the elevation of blood glucose at 30 and $60 \mathrm{~min}$ of IPGTT (fig. 4b). The alteration of glycemia prompted us to examine whether Oxt affects insulin release from pancreatic islets. Ten minutes after IPGTT, plasma insulin concentration was significantly increased $(2.19 \pm 0.26$ $\mathrm{ng} / \mathrm{ml})$, compared with the saline-injected group (0.93 \pm $0.12 \mathrm{ng} / \mathrm{ml}$; fig. $4 \mathrm{~d})$. Incubation of isolated islets with 10 nM Oxt-enhanced insulin secretion in the stimulatory (20 $\mathrm{mM})$, but not in the low (2 $\mathrm{mM})$ and basal ( $5 \mathrm{mM})$ glucose conditions (fig. 4e).

\section{Discussion}

It has recently been reported that nasal administration of Oxt reduces BW in obese humans [17]. To the best of our knowledge, the present study is the first to show the effect of nasal Oxt in reducing food intake in rodents without affecting locomotor activity or blood glucose level. This may suggest a selective anorexigenic action of nasal treatment of Oxt.

Neumann et al. [19] reported the increase of brain and plasma Oxt concentrations after nasal administration in rats and mice. The dose $(12 \mu \mathrm{g} / 10 \mu \mathrm{l})$ and application method used in their study are almost identical to those used here $(10 \mu \mathrm{g} / 10 \mu \mathrm{l})$. Hence, we speculate that Oxt levels in the brain and plasma may have been increased after nasal administration of Oxt in the present study. Nasal administration of Oxt may lead to its inhalation into the lungs [20], thereby increasing the plasma Oxt level. If so, pharmacokinetics of Oxt maybe similar between nasal and IP applications. Hence, the similar anorexigenic effects of 

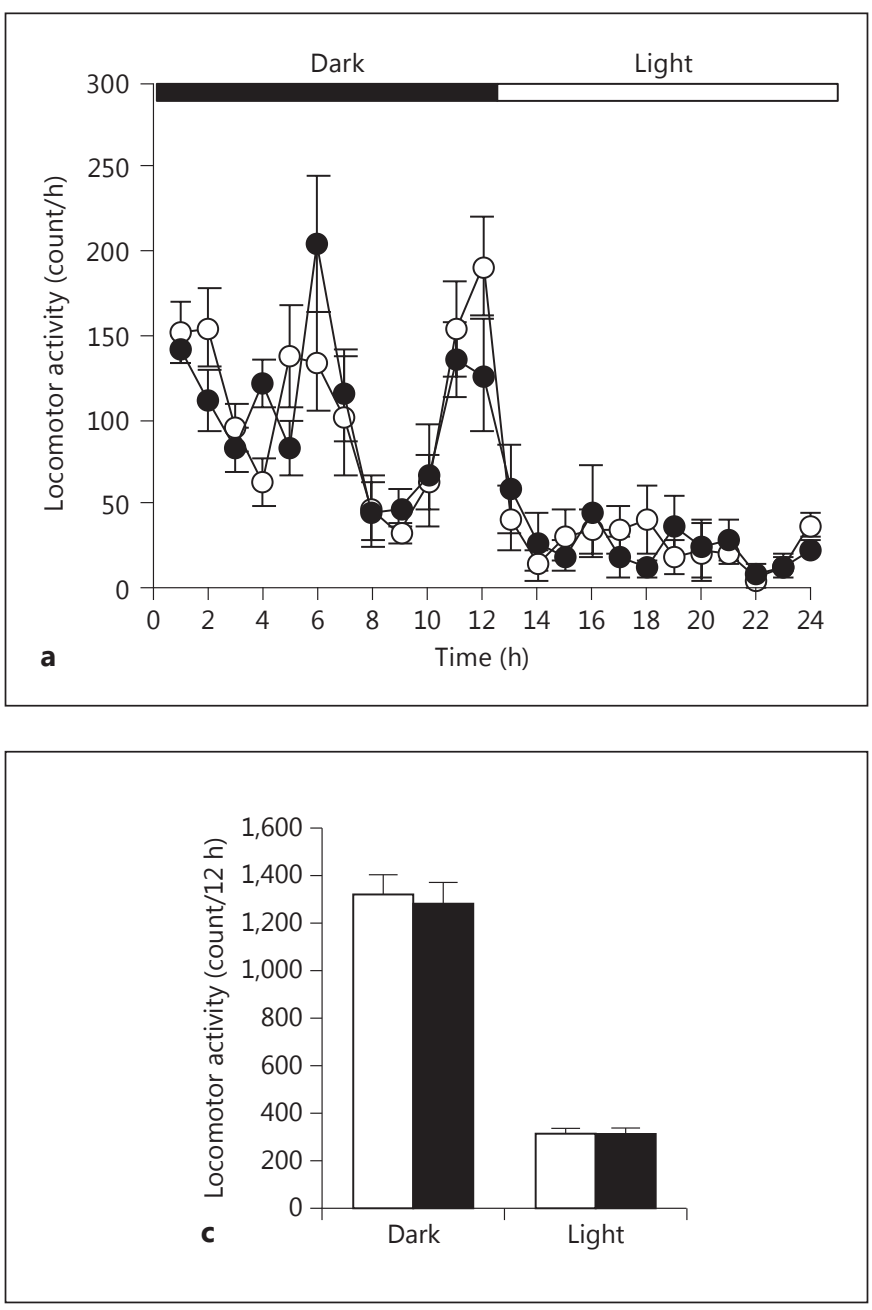

Fig. 2. Effects of nasal and IP injections of Oxt on locomotor activity in mice. a, b Locomotor activity for every $1 \mathrm{~h}$ during the 24hour period after nasal administration of $10 \mu \mathrm{g}$ Oxt ( $\mathrm{n}=7-8$; $\mathbf{a})$ and IP administration of $400 \mu \mathrm{g} / \mathrm{kg}$ Oxt $(\mathrm{n}=4 ; \mathbf{b})$. Nasal and IP administration of Oxt was performed just before the dark phase. Empty and full circles indicate the control and Oxt-injected groups, respectively. Differences were assessed by repeated mea-

nasal and IP Oxt treatments shown in the present study may have been mediated largely by the increase of peripheral Oxt concentration. In addition, Oxt is reported to cross the blood-brain barrier (BBB) from the blood to the brain by $0.05 \%$ [21]. The permeation of Oxt through the $\mathrm{BBB}$ depends on the concentration of Oxt in vessels. Since our study used a high dose of Oxt via nasal and IP routes, a significant amount of the peptide may have reached the central nervous system by crossing the $\mathrm{BBB}[3,22]$.

On the other hand, this study has shown that both nasal and IP administration of Oxt induces c-Fos in PVN,
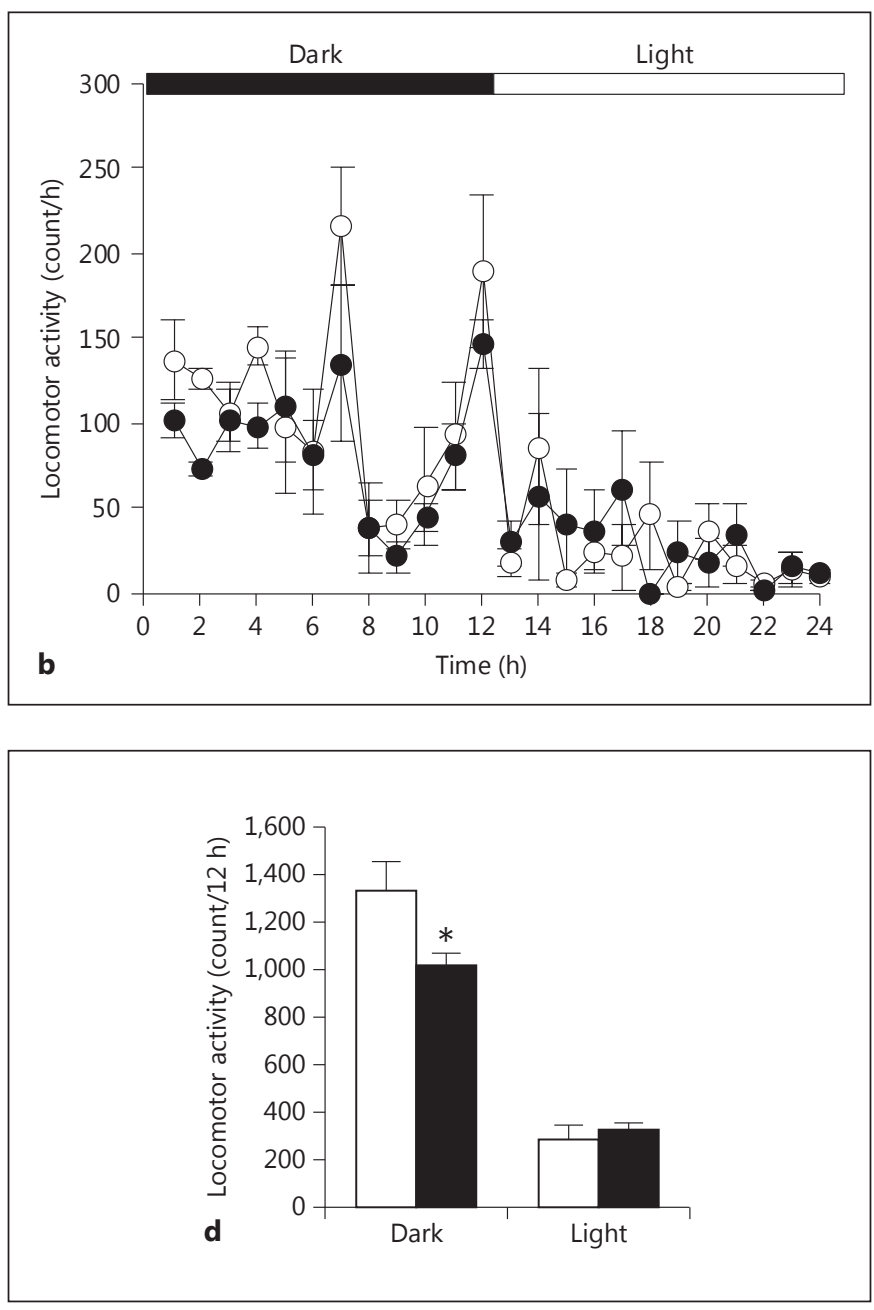

sures of two-way ANOVA with Oxt treatment and time as factors. Post hoc multiple comparisons were made using Tukey's test. $* \mathrm{p}<0.05$. c, d Cumulative locomotor activity after nasal (c) and IP administration of Oxt (d). Open and solid bars indicate the control and Oxt-injected groups. Differences were assessed by Student's t test.

$\mathrm{AP}$, and DMNV. In addition, only IP administration of Oxt induces c-Fos in ARC and NTS.

In apparent discrepancy with our results, nasal administration of Oxt $(1 \mu \mathrm{g})$ has been reported to have no effect on c-Fos-ir neurons in rats [23]. However, this discrepancy may be explained by the dose of Oxt. In the study by Ludwig et al. [23], the dose of Oxt used was $1 \mu \mathrm{g}$ for rats (BW: 250-300 g). In the present study, it was $1 \mu \mathrm{g}$ for mice (BW: 25-30 g), a factor 10 times higher when corrected for BW. Oxt doses that induced c-Fos in the present study correspond to 1-100 times of the total Oxt content of ap- 

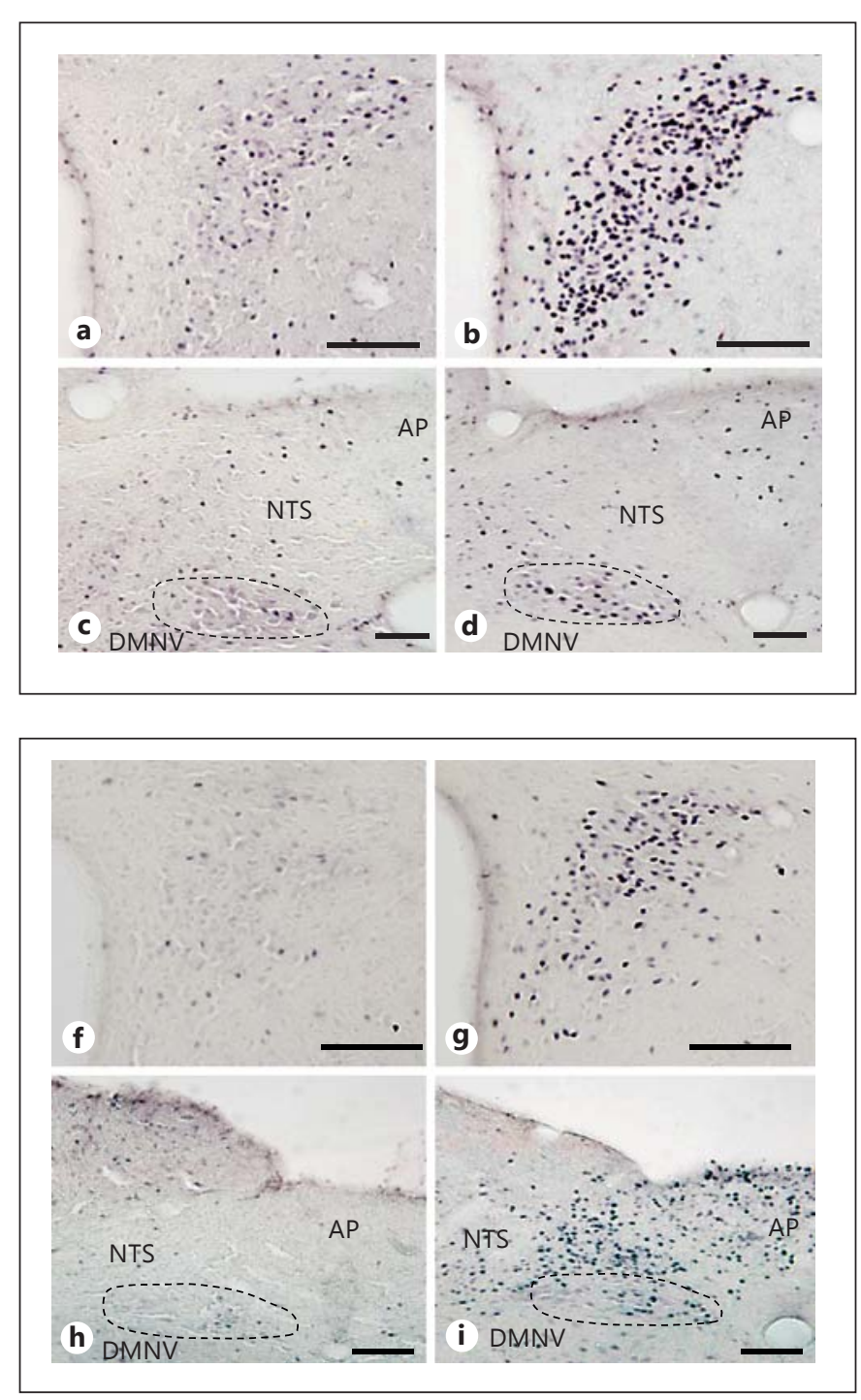

Fig. 3. c-Fos-ir neurons after nasal and IP Oxt injection. a-d Microphotographs of c-Fos-ir neurons in the PVN $(\mathbf{a}, \mathbf{b})$ and dorsal vagal complex (c, d) after nasal administration of saline $(\mathbf{a}, \mathbf{c})$ or $1 \mu \mathrm{g}$ Oxt (b, d). e Number of c-Fos-ir neurons after nasal administration of Oxt in feeding-related areas in the hypothalamus and brain stem $(\mathbf{n}=4)$. $\mathbf{f}-\mathbf{i}$ Microphotographs of c-Fos-ir neurons in

proximately $150 \mathrm{ng}$ in the mouse pituitary [24]. Hence, it is suggested that a relatively high dose of nasal Oxt is required to induce c-Fos expression. In addition, the present study used a higher dose of Oxt (approx. $10 \mu \mathrm{g}$ nasal, approx. $400 \mu \mathrm{g} / \mathrm{kg} \mathrm{IP)} \mathrm{than} \mathrm{that} \mathrm{used} \mathrm{in} \mathrm{a} \mathrm{human} \mathrm{study}$ (approx. $48 \mu \mathrm{g}$ nasal) [17]. The different dose requirement between humans and rodents is possibly due to different anatomical structures and/or to the different approach for nasal Oxt administration between humans
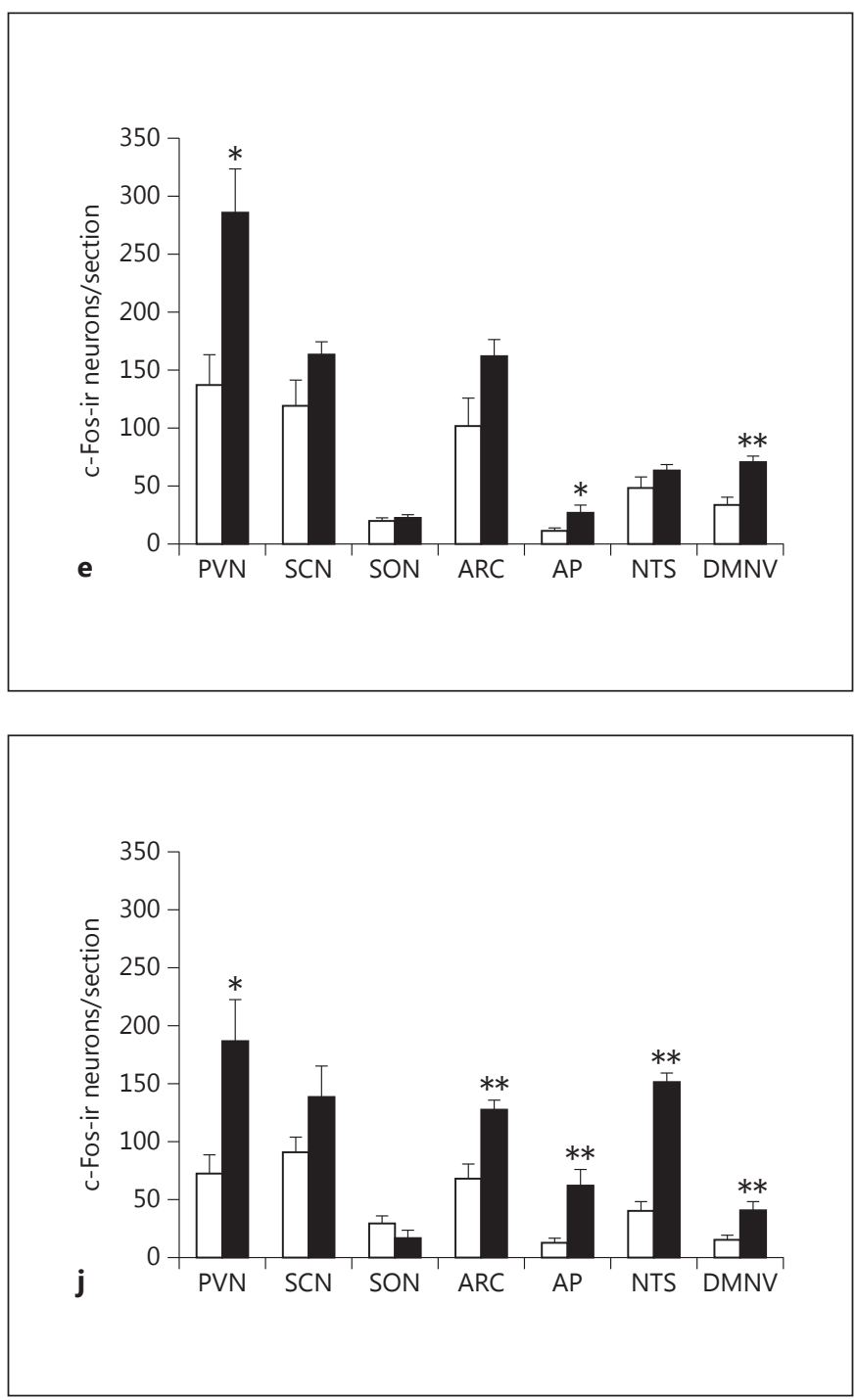

$\operatorname{PVN}(\mathbf{f}, \mathbf{g})$ and dorsal vagal complex $(\mathbf{h}, \mathbf{i})$ after IP injection of saline $(\mathbf{f}, \mathbf{h})$ or $400 \mu \mathrm{g} / \mathrm{kg}$ Oxt $(\mathbf{g}, \mathbf{i})$. j Number of c-Fos-ir neurons after IP injection of Oxt in feeding-related areas $(n=4)$. Scale bars $=100 \mu \mathrm{m}$. Differences were assessed for significance by Student's t test. ${ }^{*} \mathrm{p}<0.05,{ }^{* *} \mathrm{p}<0.01$. SCN $=$ Suprachiasmatic nucleus; $\mathrm{SON}=$ supraoptic nucleus.

(vaporizer or nasal spray) and mice (dropping the Oxtcontaining solution on the nose by a pipette).

In this study, Oxt administration via the nasal versus the IP route increased the number of c-Fos-ir neurons in both common and distinct brain areas. Both nasal and IP administration of Oxt increased c-Fos-ir neurons in the PVN, AP, and DMNV. The increase in the PVN may underlie the common action of nasal and IP Oxt administration to suppress food intake, since PVN is recognized as 

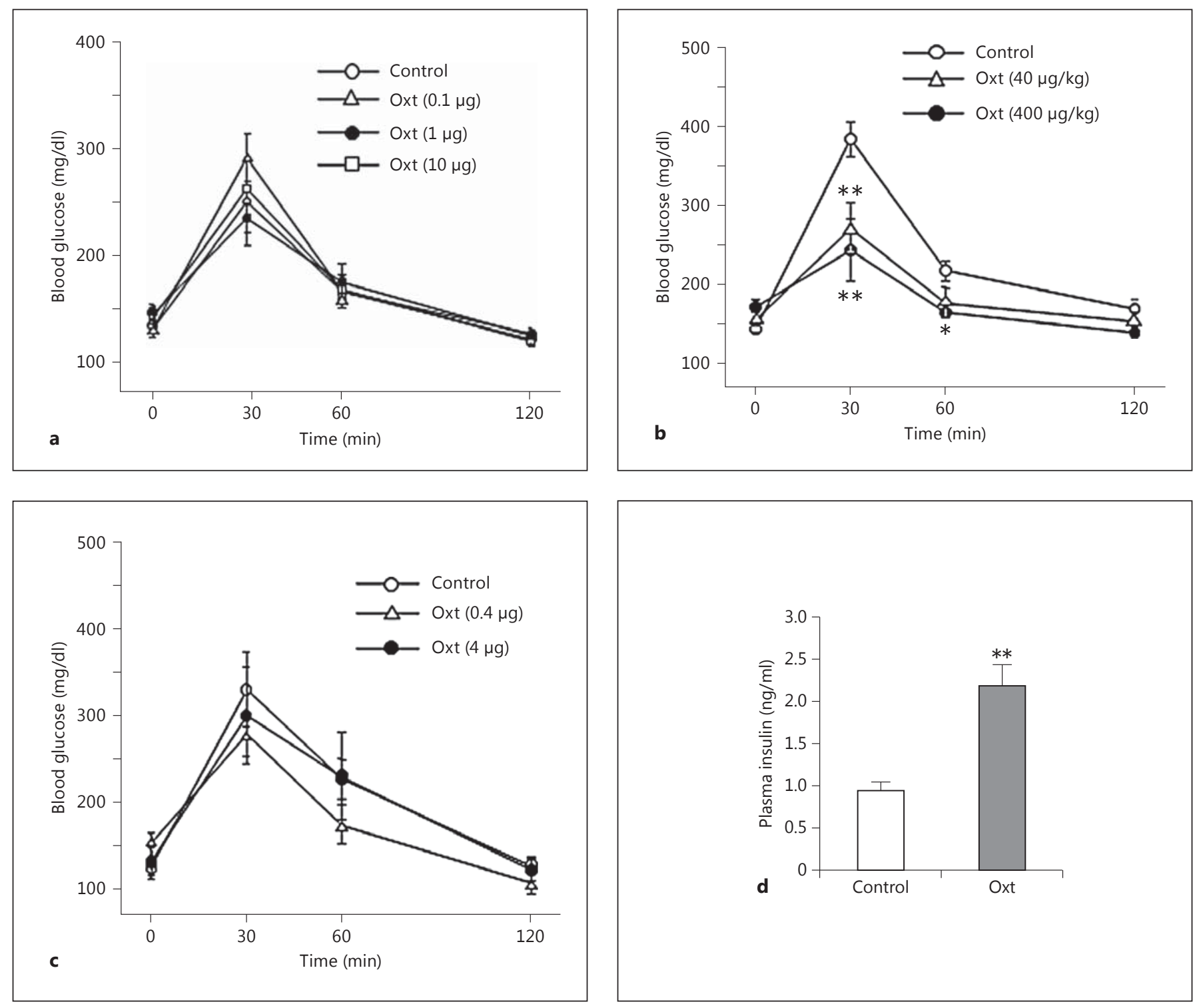

Fig. 4. Effect of Oxt on blood glucose levels in glucose tolerance test and insulin levels. a-c Blood glucose profiles in IPGTT after Oxt administration via nasal $(0.1,1$, and $10 \mu \mathrm{g} ; \mathrm{n}=6-11$; a), IP (40 and $400 \mu \mathrm{g} / \mathrm{kg} ; \mathrm{n}=5 ; \mathbf{b})$ and $\mathrm{icv}$ administration $(0.4$ and $4 \mu \mathrm{g} ; \mathrm{n}=$ $5-8$; c). Differences were assessed by repeated measures two-way ANOVA with Oxt treatment and time as factors. For each time point, post hoc multiple comparisons were made using Tukey's test. ${ }^{*} \mathrm{p}<0.05,{ }^{* *} \mathrm{p}<0.01$ versus control. d Plasma insulin levels after IP administration of Oxt. ${ }^{* *} \mathrm{p}<0.01$. e Insulin secretion from isolated pancreatic islets under static incubation with low $(2 \mathrm{mM})$, basal (5 mM), and stimulatory $(20 \mathrm{mM})$ glucose concentrations. Open bars indicate the control group $(\mathrm{n}=11)$ and solid bars indicate the Oxt-treated group $(n=11)$. Differences were assessed for significance by Student's t test. ${ }^{*} \mathrm{p}<0.05$. 
an integrative center for feeding and energy metabolism $[25,26]$. The neurons in the ARC of the hypothalamus sense various nutrients and hormones and convey neural information to the neurons in PVN, where peripheral and central information is integrated [27]. In contrast, only IP Oxt increased c-Fos-ir neurons in the ARC and NTS. Several pathways may be considered for the common and distinct brain signalling by nasal and IP Oxt. First, given that the cribriform plate is the only structure separating the nasal cavity from the olfactory bulb in rodents, nasal Oxt administration may activate the Oxt-R present in the olfactory bulb $[28,29]$. Second is the route mediated by the AP. c-Fos-ir neurons were increased in the AP after both IP and nasal application of Oxt. The AP is known to have a leaky $\mathrm{BBB}$, and the neurons in the AP project to NTS neurons, which in turn project to the ARC and PVN [30]. Hence, it is possible that nasal and IP injections of Oxt exert their effects on feeding via activating neurons in AP. However, in this study Oxt increased c-Fos-ir neurons in the NTS only after IP injection and not after nasal administration. This may indicate that neural signal transduction through the AP to NTS projection after nasal application of Oxt was not intensive enough to induce c-Fos-ir neurons in the NTS. Third is the route via vagal afferents. In this study, only IP administration of Oxt increased the number of c-Fos-ir neurons in the NTS, where the vagal afferent neurons terminate [31]. Oxt- $\mathrm{R}$ is widely expressed in the peripheral tissues including the nodose ganglion [32]. Hence, it is possible that IP-administered Oxt interacts with Oxt-R on the vagal afferent nodose ganglion neurons to signal to the NTS.

Regarding the reduced locomotor activity found only with IP administration of Oxt, the underlying mechanisms could be due to the passage of Oxt from the blood to the brain, thereby activating responsible brain regions, or to the consequences of the peripheral actions of very high levels of Oxt following IP administration. It is important to note that a high peripheral Oxt concentration can also activate vasopressin receptors that have been reported to regulate locomotor activity [33]. Further studies are required to elucidate the mechanism underlying the effect of IP Oxt administration on locomotor activities.

In the present study, nasal and IP administration of Oxt increased the number of c-Fos-ir neurons in the PVN of male mice, suggesting a possible peripheral-central Oxt axis. The result is in agreement with a recent report that the IP administration of Oxt activates PVN Oxt neurons in male rats [34]. Negative feedback effects of Oxt on Oxt neurons were reported under particular conditions in vir- gin and pregnant female rats, in contrast to positive feedback effects in male and lactating female rats [35-37]. These reports suggest that Oxt activates Oxt neurons in male rodents. Hence, our results could reflect the direct effects of exogenously administered Oxt on PVN Oxt neurons or indirect effects mediated by the nasal/IP Oxtactivated neurons that project to the PVN Oxt neurons.

We have previously reported that chronic Oxt treatment ameliorates the impaired glucose tolerance in highfat diet-induced obese mice [15]. Since chronic Oxt treatment also ameliorated obesity, the improvement of glucose tolerance by Oxt could be due to its direct effect on glucose metabolism or secondary to the amelioration of obesity. In the present study, IP administration of $40 \mu \mathrm{g} /$ $\mathrm{kg}$ Oxt induced a small effect on food intake but markedly decreased blood glucose levels at $30 \mathrm{~min}$ of IPGTT. Furthermore, Oxt elevated plasma insulin level in vivo and enhanced glucose-induced insulin secretion in isolated islets in vitro. These results taken together suggest that IP-administered Oxt substantially reaches the pancreas and directly interacts with islet $\beta$-cells to enhance glucose-induced insulin release, thereby improving glucose tolerance. Insulin, released postprandially [38], might enter the brain and inhibit food intake [27]. It was also reported that insulin activates vagal afferent neurons, which could be linked to the regulation of food intake [39]. Therefore, it is possible that insulin secretion induced by IP Oxt may partly contribute to the anorexigenic effect of IP Oxt.

In our study, nasal Oxt administration failed to affect glucose tolerance in mice. In dogs, in contrast, nasal Oxt at a similar dose $(3-4 \mu \mathrm{g} / \mathrm{kg})$ was reported to increase plasma insulin and glucagon levels [40]. Also, in humans, nasal administration of Oxt (24 IU) attenuated the elevated blood glucose following the test buffet [17]. The apparent discrepancy in the effect of nasal Oxt on glucose homeostasis could be due to species differences or methodology of treatment as stated above. The nasally administered Oxt might be more easily transferred to the periphery in dogs and humans than in mice.

In order to assess the direct cerebral effect of Oxt on glucose metabolism, we performed the IPGTT after icv injection of Oxt. icv injection of Oxt $(0.4,4 \mu \mathrm{g} / \mathrm{mice}) \mathrm{had}$ no effect on glucose metabolism. Although regulation of glucose metabolism through the brain is established, whether this process may be modulated by Oxt is not clear $[41,42]$. Our results suggest that Oxt in the brain has little effect on glucose metabolism under the experimental conditions studied here.
Maejima/Rita/Santoso/Aoyama/Hiraoka/ Nishimori/Gantulga/Shimomura/Yada 
When considering the physiological aspect of Oxt on females, it shows different effects on food intake under different conditions. In the preparturition period, Oxt secretion is very low in order to reduce the risk of preterm delivery and to prevent the loss of the accumulating neurophysial Oxt store [43], which is accompanied by an increase in food intake in both mice and rats $[42,44]$. During the parturition and lactation periods, in spite of the extensive activation of Oxt neurons, food intake is dramatically elevated [44]. It is suggested that the involvement of Oxt in feeding behavior is unique in these periods when the energy intake and expenditure are in different states from those in male or virgin animals.

Recent reports have shown that Oxt treatment reduces reward-driven food intake [17]. It is well known that the reward process, including feeding, sexual [45] and social [46] rewards, is related to the mesocorticolimbic dopamine system in the brain $[47,48]$. Oxt appears to impact this system [47], in which dopamine is one of the neurotransmitters playing a major role in addiction. It has recently been reported that Oxt modulates the negative aspect of addiction [49] and that Oxt treatment can attenuate the addictive cocaine-seeking behavior in humans [50]. Hyperphagia is considered an addictive behavior for food. The common mechanisms for hyperphagia and addiction have been reported [51]. Therefore, Oxt as a neuropeptide may serve to keep the reward system at a moderate level.

In conclusion, nasal and IP administration of Oxt have similar anorexigenic effects. However, nasal administra- tion of Oxt may have a lesser effect on the peripheral tissue, including pancreatic $\beta$-cells, compared to IP administration. Hence, nasal administration may have the advantage of activating specific brain regions without affecting glucose tolerance and locomotion.

\section{Acknowledgements}

The authors appreciate Dr. Tertia Softley (Oxford UK) for critically reading this paper. This work was supported by Grant-inAid for Scientific Research (C) (24591341), Kowa Life Science Foundation, and a research grant of the Astellas Foundation for Research on Metabolic Disorders to Y.M. This study was partly supported by Grant-in-Aid for Scientific Research (B) (23390044), for Challenging Exploratory Research $(22659044,24659101)$, and for Scientific Research on Innovative Areas (23115715) from the Japan Society for the Promotion of Science (JSPS), Strategic Research Program for Brain Sciences (10036069) by the Ministry of Education, Culture, Sports, Science and Technology of Japan (MEXT), MEXT-Supported Program for the Strategic Research Foundation at Private Universities 2011-2015 (Cooperative Basic and Clinical Research on Circadian Medicine) and 2013-2017, a Grant-in-Aid from Health Labor Sciences Research Grants from the Ministry of Health, Labor, and Welfare, Japan, grants from the Japan Diabetes Foundation, Takeda Science Foundation, and Uehara Memorial Foundation, and Insulin Research Award from Novo Nordisk to T.Y. This study was subsidized by the JKA Foundation through its promotion funds from KEIRIN RACE to T.Y.

\section{Disclosure Statement}

The authors have no conflicts of interest to disclose.

\section{References}

1 Kiss A, Mikkelsen JD: Oxytocin - anatomy and functional assignments: a minireview. Endocr Regul 2005;39:97-105.

$\checkmark 2$ Donaldson ZR, Young LJ: Oxytocin, vasopressin, and the neurogenetics of sociality. Science 2008;322:900-903.

-3 Neumann ID, Landgraf R: Balance of brain oxytocin and vasopressin: implications for anxiety, depression, and social behaviors. Trends Neurosci 2012;35:649-659.

-4 Kosfeld M, Heinrichs M, Zak PJ, Fischbacher, Fehr E: Oxytocin increases trust in humans. Nature 2005;435:673-676.

5 Feldman R, Weller A, Zagoory-Sharon O, Levine A: Evidence for a neuroendocrinological foundation of human affiliation: plasma oxytocin levels across pregnancy and the postpartum period predict mother-infant bonding. Psychol Sci 2007;18:965-970.
-6 Jin D, Liu HX, Hirai H, Torashima T, Nagai T, Lopatina O, Shnayder NA, Yamada K, Noda M, Seike T, Fujita K, Takasawa S, Yokoyama S, Koizumi K, Shiraishi Y, Tanaka S, Hashii M, Yoshihara T, Higashida K, Islam MS, Yamada N, Hayashi K, Noguchi N, Kato I, Okamoto H, Matsushima A, Salmina A, Munesue T, Shimizu N, Mochida S, Asano M, Higashida H: CD38 is critical for social behaviour by regulating oxytocin secretion. Nature 2007;446:42-45.

7 Maejima Y, Sedbazar U, Suyama S, Kohno D, Onaka T, Takano E, Yoshida N, Koike M, Uchiyama Y, Fujiwara K, Yashiro T, Horvath TH, Dietrich MO, Tanaka S, Dezaki K, Oh-I S, Hashimoto K, Shimizu H, Nakata M, Mori M, Yada T: Nesfatin-1-regulated oxytocinergic signalling in the paraventricular nucleus causes anorexia through a leptin-independent melanocortin pathway. Cell Metab 2009; 10:355-365.
-8 Olson BR, Drutarosky MD, Chow MS, Hruby VJ, Stricker EM, Verbalis JG: Oxytocin and oxytocin agonist administered centrally decrease food intake in rats. Peptides 1991;12: 113-118.

-9 Arletti R, Benelli A, Bertolini A: Oxytocin inhibits food intake and fluid intake in rats. Physiol Behav 1990;48:825-830.

-10 Takayanagi Y, Kasahara Y, Onaka T, Takahashi N, Kawada T, Nishimori K: Oxytocin receptor-deficient mice developed late-onset obesity. Neuroreport 2008;9:951-955.

11 Camerino C: Low sympathetic tone and obese phenotype in oxytocin-deficient mice. Obesity (Silver Spring) 2009;17:980-984.

12 Ogden CL, Kit BK, Carroll MD, Flegal KM: Prevalence of obesity and trends in body mass index among US children and adolescents, 1999-2010. JAMA 2012;307:483-490. 
13 Bardou M, Barkun AN, Martel M: Obesity and colorectal cancer. Gut 2013;62:933-947.

-14 Suplicy H, Boguszewski CL, Dos Santos CM, do Desterro de Figueiredo M, Cunha DR, Radominski R: A comparative study of five centrally acting drugs on the pharmacological treatment of obesity. Int J Obes (Lond) 2014; 38:1097-1103.

-15 Maejima Y, Iwasaki Y, Yamahara Y, Kodaira M, Sedbazar U, Yada T: Peripheral oxytocin treatment ameliorates obesity by reducing food intake and visceral fat mass. Aging (Albany NY) 2011;3:1169-1177.

-16 Zhang H, Wu C, Chen Q, Chen X, Xu Z, Wu J, Cai D: Treatment of obesity and diabetes using oxytocin or analogs in patients and mouse models. PLoS One 2013;8:1-11.

${ }_{17}$ Ott V, Finlayson G, Lehnert H, Heitmann B, Heinrichs M, Born J, Hallschmid M: Oxytocin reduces reward-driven food intake in humans. Diabetes 2013;62:3418-3425.

-18 Shimomura K, Galvanovslis J, Goldsworthy M, Hugill A, Kaizak S, Lee A, Meadows N, Quwailid MM, Rydström J, Teboul L, Ashcroft F, Cox RD: Insulin secretion from betacells is affected by deletion of nicotinamide nucleotide transhydrogenase. Methods Enzymol 2009;457:451-480.

19 Neumann ID, Maloumby R, Beiderbeck DI, Lukas M, Landgraf R: Increased brain and plasma oxytocin after nasal and peripheral administration in rats and mice. Psychoneuroendocrinology 2013;38:1985-1993.

-20 Ramos L, Hicks C, Caminer A, McGregor IS: Inhaled vasopressin increases sociability and reduces body temperature and heart rate in rats. Psychoneuroendocrinology 2014;46:4651.

21 Mens WB, Witter A, Van Wismersm Greidanus TB: Penetration of neurophyseal hormones from plasma into cerebrospinal fluid (CSF): half-times of disappearance of these neuropeptides from CSF. Brain Res 1983;262: 143-149.

22 Banks WA, Kastin AJ: Passage of peptides across the blood-brain barrier: pathophysiological perspectives. Life Sci 1996;59:19231943.

23 Ludwig M, Tobin VA, Callahan MF, Papadaki E, Becker A, Engelmann M, Leng G: Intranasal application of vasopressin fails to elicit changes in brain immediate early gene expression. Neural activity and behavioral performance of rats. J Neuroendocrinol 2013;25: 655-667.

-24 Bridges TE, James NV: The hypothalamoneurohypophysial system of native Australian desert rodents. The vasopressin and oxytocin contents of hypothalamus and posterior pituitary of Notomys alexis and Pseudomys australis compared with those of the laboratory rat and mouse in different states of water balance. Aust J Exp Biol Med Sci 1982;60: 265-283.
25 Swanson LW, Sawchenko PE: Hypothalamic integration: organization of the paraventricular and supraoptic nuclei. Annu Rev Neurosci 1983;6:269-324.

26 Xu Y, Wu Z, Sun H, Zhu Y, Kim ER, Lowell BB, Arenkiel BR, Xu Y, Tong Q: Glutamate mediates the function of melanocortin receptor 4 on Sim 1 neurons in body weight regulation. Cell Metab 2013;18:860-870.

27 Schwartz MW, Woods SC, Porte D Jr, Seeley RJ, Baskin DG: Central nervous system control of food intake. Nature 2000;404:661-671.

28 Putheti RR, Patil MC, Obire O: Nasal drug delivery in pharmaceutical and biotechnology: present and future. J Sci Technol 2009;4:1-21.

29 Gould BR, Zingg HH: Mapping oxytocin receptor gene expression in the mouse brain and mammary gland using an oxytocin receptor-lacZ reporter mouse. Neuroscience 2003; 122:155-168.

-30 Stein MK, Loewy AD: Area postrema projects to FoxP2 neurons of the pre-locus and parabranchial nuclei: brainstem sites implicated in sodium appetite regulation. Brain Res 2010;1359:116-127.

31 Browning KN, Travagli RA: Plasticity of vagal brainstem circuits in the control of gastrointestinal function. Auton Neurosci 2011;161: 6-13.

32 Welch MG, Tamir H, Gross KJ, Chen J, Anwar M, Gershon MD: Expression and developmental regulation of oxytocin (OT) and oxytocin receptors (OTR) in the entric nervous system (ENS) and intestinal epithelium. J Comp Neurol 2009;512:265-270.

33 Cormier HC, Della-Maggiore V, Karatsoreos IN, Koletar MM, Ralph MR: Suprachiasmatic vasopressin and the circadian regulation of voluntary locomotor behavior. Eur J Neurosci 2015;41:79-88.

34 Zhang G, Cai D: Circadian intervention of obesity development via resting-stage feeding manipulation or oxytocin treatment. Am J Physiol Endocrinol Metab 2011;301:E1004E1012.

35 Moos F, Freund-Mercier MJ, Guerné Y, Guerné JM, Stoeckel ME, Richard P: Release of oxytocin and vasopressin by magnocellular nuclei in vitro: specific facilitatory effect of oxytocin on its own release. J Endocrinol 1984;102:63-72.

-36 Neumann I, Koehler E, Landgraf R, SummyLong J: An oxytocin receptor antagonist infused into the supraoptic nucleus attenuates intranuclear and peripheral release of oxytocin during suckling in conscious rats. Endocrinology 1994;134:141-148.

37 Kawarabayashi T, Kuriyama K, Nakashima T, Kiyohara T, Sugimori H: Oxytocin modulates oxytocin neurons in the paraventricular nuclei of female rats throughout pregnancy and parturition. Am J Obstet Gynecol 1993;168: 969-974.
8 Cummings DE, Purnell JQ, Frayo RS, Schmidova K, Wisse BE, Weigle DS: A preprandial rise in plasma ghrelin levels suggests a role in meal initiation in humans. Diabetes 2001;50: 1714-1719.

39 Iwasaki Y, Shimomura K, Kohno D, Dezaki K, Ayush EA, Nakabayashi H, Kubota N, Kadowaki T, Kakei M, Nakata M, Yada T: Insulin activates vagal afferent neurons including those innervating pancreas via insulin cascade and $\mathrm{Ca}^{2+}$ influx: its dysfunction in IRS2KO mice with hyperphagic obesity. PLoS One 2013;8:e67198.

40 Altszuler N, Hampshire J: Intranasal instillation of oxytocin increases insulin and glucagon secretion. Proc Soc Exp Biol Med 1981; 168:123-124.

41 Schwartz MW, Seeley RJ, Tschop MH, Woods SC, Morton GJ, Myers MG, D’Alesso D: Cooperation between brain and islet in glucose homeostasis and diabetes. Nature 2013;503: 59-66.

42 Björkstrand E, Eriksson M, Uvnäs-Moberg K: Evidence of a peripheral and a central effect of oxytocin on pancreatic hormone release in rats. Neuroendocrinology 1996;63: 377-383.

43 Douglas AJ, Johnstone LE, Leng G: Neuroendocrine mechanisms of change in food intake during pregnancy: a potential role for brain oxytocin. Physiol Behav 2007;24:352-365.

44 Makarova EN, Kochubei ED, Bazhan NM: Regulation of food consumption during pregnancy and lactation in mice. Neurosci Behav Physiol 2010;40:263-267.

45 Argiolas A, Melis MR: The role of oxytocin and the paraventricular nucleus in the sexual behaviour of male mammals. Physiol Behav 2004;83:309-317.

46 Young KA, Liu Y, Gobrogge KL, Wang H, Wang Z: Oxytocin reverses amphetamine-induced deficits in social bonding: evidence for an interaction with nucleus accumbens dopamine. J Neurosci 2014;34:8499-8506.

47 Love TM: Oxytocin, motivation and the role of dopamine. Pharmacol Biochem Behav 2014;119:49-60.

48 Gimpl G, Fahrenholz F: The oxytocin receptor system: structure, function, and regulation. Physiol Rev 2001;81:629-683.

49 McGregor IS, Bowen MT: Breaking the loop: oxytocin as a potential treatment for drug addiction. Horm Behav 2012;61:331-339.

-50 Morales-Rivera A, Hernández-Burgos MM Martínez-Rivera A, Pérez-Colón J, Rivera R, Montalvo J, Rodríguez-Borrero E, Maldonado-Vlaar CS: Anxiolytic effects of oxytocin in cue-induced cocaine seeking behavior in rats. Psychopharmacology (Berl) 2014;231:41454155.

51 Kenny PJ: Common cellular and molecular mechanisms in obesity and drug addiction. Nat Rev Neurosci 2011;12:638-651. 